\title{
ASCARIDÍASE, HIMENOLEPÍASE, AMEBÍASE E GIARDÍASE: UMA ATUALIZAÇÃO
}

\author{
Francisco Patricio de Andrade Júnior ${ }^{1 *}$, Thiago Willame Barbosa Alves², \\ Vanessa Santos de Arruda Barbosa ${ }^{3}$ \\ ${ }^{1}$ Doutorando, Programa de Pós-Graduação em Produtos Naturais e Sintéticos Bioativos, \\ Centro de Ciências da Saúde, Universidade Federal da Paraíba, João Pessoa-PB. \\ ${ }^{2}$ Farmacêutico pela Universidade Federal de Campina Grande. \\ ${ }^{2}$ Prof $^{\mathrm{a}}$. Dra . Unidade Acadêmica de Saúde, Universidade Federal de Campina Grande, Cuité, \\ PB, Brasil. \\ Email para correspondência: juniorfarmacia.ufcg@outlook.com
}

\begin{abstract}
Resumo
As parasitoses intestinais são doenças que prevalencem em países subdesenvolvidos como o Brasil e, se caracterizam por tratarem-se de um importante problema de saúde pública. Ademais, na literatura há grande escassez de estudos que abordem sobre alguns tipos de enteroparasitoses como a ascaridíase, amebíase, giardíase e himenolepíase. Objetivou-se realizar uma revisão de literatura acerca das parasitoses ascaridíase, himenolepíase, amebíase e giardíase e seus respectivos agentes etiológicos. Revisão de literatura do tipo narrativa, realizada a partir de artigos publicados entre 2010 a 2019. Identificou-se que as enteroparasitoses analisadas são adquiridas sobretudo, a partir de alimentos e água contaminados ou até mesmo por meio da ingestão de insetos (himenolepíase) ou de alimentos que tiveram contato com o solo (ascaridíase). A sintomatologia é bastante semelhante, de forma que há a necessidade da realização de diagnóstico laboratorial que envolvam métodos de sedimentação e centrifugação. O tratamento é feito, exclusivamente, por meio de fármacos atrelado a medidas prófiláticas para se evitar a reinfecção. Assim, este estudo pode servir para embasar outras pesquisas que tenham as parasitoses intestinais como foco centralizador e também atuar como possível material didático para a atualização de profissionais da saúde.
\end{abstract}

Palavras-chave: Parasitoses intestinais, Ascaris lumbricoides, Hymenolepis nana, Entamoeba histolytica/E.dispar, Giardia lamblia.

\section{Abstract}

The intestinal parasites are diseases that prevail in underdeveloped countries such as Brazil and are characterized by being an important public health problem. In addition, in the literature there is a great shortage of studies that deal with some types of enteroparasitoses such as ascaridiasis, amoebiasis, giardiasis and hymenolepiiasis. The objective was to conduct a literature review about the parasites ascaridiasis, hymenolepiasis, amebiasis and giardiasis and respective etiological agents. Literature review of the narrative type, carried out from articles published between 2010 and 2019. It was observed that the enteroparasitoses analyzed are acquired mainly from contaminated food and water or even through the ingestion of insects (hymenolepiasis) or food that had contact with the soil (ascaridiasis). The symptomatology is quite similar, so there is a need for 
laboratory diagnosis involving sedimentation and centrifugation methods. The treatment is done exclusively by means of drugs tied to prophylactic measures to avoid reinfection. Thus, this study may serve as a basis for other researches that have intestinal parasitoses as a centralizing focus and also act as possible didactic material for updating health professionals.

Keywords: Intestinal parasites, Ascaris lumbricoides, Hymenolepis nana, Entamoeba histolytica/E.dispar, Giardia lamblia.

\section{Introdução}

Nas últimas décadas o Brasil tem passado por modificações que têm ocasionado em melhor qualidade de vida para sua população, entretanto as parasitoses ainda são endêmicas em diversas regiões do país (BELO et al., 2012).

As ocorrências das infecções parasitárias estão relacionadas à interação entre parasito, hospedeiro e meio ambiente, levando-se em consideração características inerentes ao indivíduo (imunidade), ao parasito (virulência e carga contaminante) e as questões culturais e sociais (costumes, saneamento, higiene pessoal, qualidade da água e alimentos) (ANDRADE et al., 2010; CHIEFFI, 2015).

Assim, é possível conceituar as parasitoses intestinais como doenças cosmopolitas causadas por protozoários e/ou helmintos que possuem uma grande prevalência em regiões tropicais (LIMA, 2011).

Diversos parasitos são comumente encontrados no Brasil e são passíveis a contaminar alimentos e causar afecções características ou até mesmo atuarem juntos, disseminando no indivíduo parasitado mais de uma parasitose. Dentre as espécies de parasitos que podem ser encontrados em alimentos e água contaminados, tem-se: Trichinella spiralis, Toxoplasma gondii, Cryptosporidium parvum, Pseudoterranova decipiens, Giardia lamblia, Ascaris lumbricoides, Trichuris trichiura, Diphyllobothrium spp., Entamoeba histolytica, Taenia saginata, Taenia solium, Fasciola hepatica, Cyclospora cayetanensis, dentre outros (NEVES, 2016).

A elevada magnitude e ampla distribuição geográfica das enteroparasitoses, aliadas às repercussões negativas que podem causar no organismo humano, têm conferido a essas infecções uma posição relevante entre os principais problemas de saúde da população (FONSECA et al., 2010). Dessa forma, no controle de doenças endêmicas, não se deve ignorar as 
parasitoses intestinais, uma vez que, o seu tratamento é simples e, se levado a toda comunidade simultaneamente, torna-se mais viável economicamente (BORGES; MARCIANO; OLIVEIRA, 2011).

O tratamento das parasitoses intestinais consiste além do emprego de antiparasitários, em medidas de educação preventiva e de saneamento básico. Em vista da dificuldade de diagnóstico específico das parasitoses e a semelhança sintomatológica apresentada, muitas vezes, são realizados tratamentos empíricos com mais de um fármaco (ANDRADE et al., 2010), reforçando a necessidade da realização de exames parasitológicos que possam, em conjunto com a anamnese médica, contribuir para um correto diagnóstico e tratamento.

Há algumas espécies como Entamoeba coli, Endolimax nana e lodamoeba butschlii que biologicamente não são capazes de causar malefícios ao ser humano, contudo sua presença em amostras fecais pode indicar fragilidade na higiene pessoal e de saneamento básico (NEVES, 2016). Por outro lado, alguns parasitos podem causar importantes prejuízos a saúde, devido sua capacidade inerente de causar desenvolvimento de doenças como a ascaridíase, himenolepíase, amebíase e giardíase, ademais observa-se uma grande escassez de estudos atuais que tragam informações relevantes acerca dessas enfermidades, sendo necessário a existência de estudos de atualização.

Assim, o presente estudo objetivou realizar uma revisão de literatura acerca das parasitoses ascaridíase, himenolepíase, amebíase e giardíase, e seus respectivos agentes etiológicos.

\section{Metodologia}

\subsection{Delineamento do estudo}

Revisão da literatura do tipo narrativa. Houve a utilização de artigos, monografias, dissertações e teses publicadas em língua portuguesa, espanhola e inglesa, utilizando-se os delimitadores e palavras-chaves: 1) Ascaridíase; 2) Ascaris lumbricoides; 3) Amebíase; 4) Entamoeba histolytica/E. dispar; 5) Giardíase; 6) Giardia lamblia; 7) Himenolepíase; 8) Hymenolepis nana; 9) 
Parasitoses intestinais; 10) Enteroparasitoses; utilizados isolados e associados em várias combinações.

\subsection{Critérios de inclusão e exclusão}

Foram incluídos estudos que em seu conteúdo trouxessem informações relevantes acerca da ascaridíase, himenolepíase, amebíase e giardíase, assim como, dos seus respectivos agentes etiológico de artigos publicados durante os anos de 2010 à 2019. Estudos que não atenderam o tempo cronológico delimitado ou que apresentavam informações acerca de outros tipos de parasitoses foram excluídos da pesquisa.

\subsection{Fontes de informação}

Os artigos foram recuperados a partir das bases de dados: Lilacs (Centro América Latina e Caribe em Ciências da Saúde), Scielo (Scientific Eletronic Library Online), PubMed, ScienceDirect e Bancos de Teses e Dissertações de Universidades Públicas.

\section{Revisão de literatura}

Para o desenvolvimento dessa revisão de literatura foram analisados 71 documentos, entretanto somente 32 foram utilizados para a construção desta pesquisa.

\subsection{Ascaridíase e Ascaris lumbricoides}

A ascaridíase, em seres humanos, é uma helmintose causada por Ascaris lumbricoides, sendo comumente evidenciada em países em que há escassez de saneamento básico adequado e precária higiene pessoal e dos alimentos, afetando principalmente populações de baixa renda, geralmente mais expostas e susceptíveis a ingesta de água e alimentos contaminados contendo o parasito (ANDRADE et al., 2010; CAVALCANTE; MELO; LIMA, 2015).

Estima-se que pelo menos 320 milhões de pessoas no mundo estão contaminadas por A. lumbricoides (MAIA; HASSUM; VALLADARES, 2015), sendo este um dos helmintos mais prevalentes em crianças da região Nordeste do Brasil (LIMA et al., 2013). 
O A. lumbricoides pode apresentar-se em três formas evolutivas distintas: adulta, larval e ovo. Em relação às características e morfologia, as formas adultas apresentam dimorfismo sexual. $O$ macho mede cerca de 20 a $30 \mathrm{~cm}$ de comprimento com coloração leitosa. A boca está localizada na extremidade anterior, seguindo-se de esôfago musculoso e intestino retilíneo. Enquanto que o reto está localizado na extremidade posterior, ademais, evidencia-se a presença de testículo filiforme, canal deferente, canal ejaculador, cloaca e dois espículos iguais. A fêmea apresenta cerca de 30 a 40 $\mathrm{cm}$. A coloração, boca e aparelho digestivos são semelhantes ao do macho, porém apresenta extremidade posterior retilínea, com presença de dois ovários filiformes, ovidutos, úteros e uma única vagina, que se exterioriza pela vulva (NEVES, 2016).

As larvas, por sua vez, podem apresentar-se como rabditoides ou filarioides, dependendo justamente da forma evolutiva e o tipo de esôfago (DOLD; HOLLAND, 2011; NEVES, 2016).

Os ovos possuem coloração branca, com cerca $50 \mu \mathrm{m}$ de comprimento por $60 \mu \mathrm{m}$ de diâmetro e apresentam cápsula espessa, em razão da membrana externa, também chamada de "membrana mamilonada", sendo composta por mucopolissacarídeos. Além dessas características, os ovos que não possuem massa germinativa são considerados inférteis (NEVES, 2016).

Este parasita pode ser adquirido através da ingesta de água e alimentos contaminados por ovos (JOURDAN et al., 2017). Contudo, inicialmente os ovos necessitam passar por períodos no solo para propiciar o completo embrionamento, devendo-se estar em ambientes com 25 a $35^{\circ} \mathrm{C}$, umidade a $70 \%$ e oxigênio abundante. Após estar embrionado, tem-se o desenvolvimento da larva L1, do tipo rabditoide, em seguida após cerca de uma semana a larva sofre muda e torna-se L2 e tem-se outra muda, tornando-se L3, larva do tipo filarioide, sento esta última a forma infectante (DOLD; HOLLAND, 2011; NEVES, 2016).

Uma vez que haja a deglutição de ovos contendo L3, estas larvas são liberadas após a eclosão do ovo e migram para regiões do ceco e cólon proximal, onde penetram na mucosa atravessando à parede intestinal, adentrando a corrente linfática e sanguínea a partir da veia mesentérica 
superior, permitindo alcançar o fígado após 18 a 24 horas do início da infecção. Depois de 2 a 3 dias, chegam ao átrio direito pela veia cava inferior ganhando os pulmões. No oitavo dia de infecção as larvas L3 sofrem muda em L4, permitindo o rompimento de capilares de forma que há a penetração no espaço alveolar, onde tem-se uma nova muda, havendo o surgimento de L5. Estas últimas larvas sobem pela árvore brônquica, passam pela tranqueia e chegam à faringe, podendo ser expelidas ou deglutidas. As que forem deglutidas atravessam o estômago sem que haja nenhum comprometimento em sua estrutura, atingindo o intestino delgado. Após 20 a 30 dias tornam-se adultas; cerca de 60 dias depois do início do processo infeccioso, os parasitos estão aptos a cópula e a oviposição (DOLD; HOLLAND, 2011; NEVES, 2016).

As formas adultas de $A$. lumbricoides, quando estão em número superior a 30 podem causar diversos danos ao organismo do hospedeiro a partir de ações espoliadoras, tóxicas e mecânicas, assim como, por meio da localização ectópica. No caso da espoliação, esta ocorre através do consumo, por parte do parasito, de grandes quantidades nutrientes. A ação tóxica, por sua vez, ocorre a partir de reações entre antígenos parasitários e anticorpos alergizantes do paciente, ocasionando em edemas, urticárias e possíveis convulsões, enquanto que nas ações mecânicas, tem-se principalmente o acometimento da parede intestinal, devido irritações causadas por danos provenientes das formas adultas, além de um possível enovelamento, seguido de obstrução intestinal (ANDRADE et al., 2010; NEVES, 2016).

A localização ectópica ocorre em pacientes com altas cargas parasitárias ou ainda devido ações irritativas como febre, alimentos condimentados e medicamentos, fazendo com que 0 parasito migre para regiões incomuns, sendo chamado, portanto de "áscaris errático". As larvas quando em grande número também podem causar danos em diversos órgãos. No fígado, é possível observar pequenos focos hemorrágicos e necrose, enquanto que nos pulmões tem-se o aparecimento de pontos hemorrágicos, devido perfurações, com formação de edemas nos alvéolos e processo inflamatório com infiltração de neutrófilos e eosinófilos. Os danos alveolares causam o surgimento de tosse, febre, dispneia, eosinofilia e bronquite; o 
escarro pode apresentar-se sanguinolento, entretanto é mais comumente visto em crianças com mau aporte nutricional e imunodepressão (NEVES, 2016).

Dessa forma, a ascaridíase pode apresentar-se de forma assintomática ou sintomática. Os principais sintomas que se manifestam são dor abdominal, diarreia, náusea e anorexia, podendo ainda haver o desenvolvimento de obstrução intestinal. Além disso, devido uma parte do ciclo biológico ocorrer no pulmão, os acometidos podem desenvolver pneumonite, hemoptise, broncoespasmo e a síndrome de Löeffler, caracterizada pela eosinofilia, ademais essa doença ainda pode induzir o desenvolvimento de comorbidades, como a asma (BRASIL, 2010; JOURDAN et al., 2017).

O diagnóstico pode ser realizado através da investigação clínica juntamente com o exame parasitológico de fezes a partir de métodos de sedimentação ou Kato- Katz, sendo este último utilizado quando se quer descobrir a carga parasitária. O tratamento, por sua vez, se dá por meio dos fármacos albendazol, mebendazol e levamizol; para casos de obstrução intestinal recomenda-se piperazina, associada a óleo mineral, antiespasmódicos e hidratação, ou ainda, uso de sonda nasogástrica e jejum associado ao mebendazol. A profilaxia se dá através do tratamento em massa de habitantes de área endêmicas, saneamento básico e educação para saúde (BRASIL, 2010).

\subsection{Himenolepíase e Hymenolepis nana}

A himenolepíase é uma helmitose, causada por Hymenolepis nana, responsável por contaminar cerca de 50 a 75 milhões de pessoas no mundo, acometendo principalmente crianças nas regiões do Mediterrâneo e América do Sul, que estão expostas a precárias situações de higiene e saneamento básico (BOURÉE, 2014; THOMPSON, 2015).

Este parasito pode ser encontrado em três formas evolutivas distintas: ovo, larva cisticercóide e verme adulto. O ovo se caracteriza por ser quase esférico, transparente e incolor, com cerca de $40 \mu \mathrm{m}$ de diâmetro e possuir dois mamelões em posições opostas, com oncosfera em seu interior, enquanto que a larva cisticercóide apresenta $500 \mu \mathrm{m}$ de diâmetro e escólex invaginado, envolvido por membrana. A forma adulta, por sua vez, mede de 3 a $5 \mathrm{~cm}$ de 
comprimento, é hermafrodita, possuindo colo e escólex. O colo tem de 100 a 200 proglotes enquanto que o escólex é composto por quatro ventosas e rosto retrátil armado com ganchos (MENEZES et al., 2016).

Em relação ao ciclo biológico, esse parasito apresenta dois distintos: um monoxênico e outro, heteroxênico. O ciclo monoxênico, não necessita da presença de um hospedeiro intermediário, enquanto que o heteroxênico necessita desse tipo de hospedeiro, que são representados principalmente por insetos (pulgas: Xenopsylla cheopis, Ctenocephalides canis, Pulex irritans, e coleópteros: Tenebrio molitor, T. obscurus e Tribolium confusum) (MENEZES et al., 2016).

No caso do ciclo monoxênico, os ovos de $H$. nana são liberados juntamente com as fezes, podendo contaminar coleções aquáticas e alimentos ou as mãos. Ao ser ingerido, esse ovo passa por um processo de semidigestão, havendo assim a liberação da oncosfera no intestino, permitindo a sua penetração nas vilosidades, se transformando em larva cisticercóide. Estas vilosidades se rompem, liberando a larva que retorna a região do lúmen intestinal, estando apta a fixar-se, por meio do escólex, no intestino do hospedeiro, permitindo, assim o desenvolvimento da forma adulta que pode permanecer de 4 a 6 semanas nesta região (THOMPSON, 2015).

Enquanto que no ciclo heteroxênico, os ovos são digeridos por larvas de insetos como carunchos de cereais ou pulgas. Ao chegar no intestino desses hospedeiros, há todo o processo de digestão e liberação da oncosfera, que evolui em larva cisticercóide, dessa forma, quando seres humanos ingerem estes insetos por meio de farinhas e cereais, principalmente, as larvas chegam ao intestino delgado, desinvaginam-se, fixam-se à mucosa e em 20 dias são vermes adultos (THOMPSON, 2015).

Com baixa carga parasitária, os acometidos apresentam-se assintomáticos, entretanto, em infecções com alto número de parasitos podese observar o surgimento de diarreia, dores abdominais, insônia, agitação, irritabilidade, má absorção e déficit de crescimento em crianças (THOMPSON, 2015; MENEZES et al., 2016).

O diagnóstico pode ser dado através do exame parasitológico de fezes, recomendando-se os métodos de sedimentação espontânea e centrífugo- 
sedimentação. $O$ tratamento dos infectados é realizado por meio de albendazol e praziquantel; medidas que envolvam educação em saúde, melhoria da higiene e saneamento básico, podem dificultar o processo de perpetuação do parasito e diminuir consideravelmente 0 número de contaminados (THOMPSON, 2015; NEVES, 2016).

\subsection{Amebíase e Entamoeba histolytica/E.dispar}

A amebíase é uma das protozooses mais comuns atualmente e apresenta-se sintomática em somente 40 a 50 milhões dos acometidos, sendo responsável por cerca de 100 mil óbitos por ano (NICHOLS, 2014; NELSON; SINGH, 2018).

Esta enfermidade é causada por um agente etiológico, pertencente à família Entamoebidae, nomeado de Entamoeba histolytica que é responsável, juntamente com a $E$. dispar, por contaminar mais de 500 milhões de pessoas no mundo (ALVADOR; STRECK, 2017; NELSON; SINGH, 2018).

Morfologicamente, E. histolytica e E. dispar não apresentam diferenças visíveis, sendo impossível diferenciá-las por meio de microscopia; nas fezes, estes agentes apresentam-se em dois estágios básicos: trofozoíto e cisto. $\mathrm{O}$ trofozoíto ou forma vegetativa, pode variar de 10 a $60 \mu \mathrm{m}$ de comprimento, apresenta forma amebóide, possuindo em seu núcleo o cariossoma central e a cromatina periférica. A forma cística desses protozoários, por sua vez, possui parede cística, 10 a $20 \mu \mathrm{m}$ de diâmetro, um a quatro núcleos e citoplasma com vacúolos de glicogênio e corpos cromatóides em forma de bastonetes (NEVES, 2016).

O homem se infecta por meio da rota fecal-oral e/ou de alimentos e água contaminados com o cisto de E. histolytica/E. dispar. Além disso, outras formas de contaminação menos comuns podem ocorrer, como: o sexo anal e oral e a partir equipamentos utilizados para procedimentos de lavagem intestinal (CHAVES; SEIXAS FILHO; DANTAS, 2010).

$O$ ciclo biológico é de tipo monoxênico e inicia-se através da ingestão de cistos maduros presentes em alimentos e água contaminados. Os cistos passam pelo estômago e resistem ao suco gástrico, entretanto ao chegarem no final do intestino delgado ou no início do intestino grosso ocorre o desencistamento, havendo a liberação do metacisto. O metacisto, por sua vez, 
passa por contínuas divisões nucleares e citoplasmáticas, dando origem a quatro e depois a oito trofozoítos que são nomeados de trofozoítos metacísticos. Estes migram para o intestino grosso, havendo processo de colonização, ficando geralmente aderidos a mucosa. Em seguida, por questões ainda não elucidadas, estas estruturas se desprendem da parede intestinal e sofrem ação de desidratação, transformando-se em pré-cistos; logo em seguida, os pré-cistos secretam a membrana cística e se transformam em cistos, inicialmente mononucleados, ocorrendo depois divisões celulares sucessivas, se transformando em cistos tetranucleares prontos para serem eliminados nas fezes normais ou formadas. Dificilmente há presença de cistos nas fezes diarréicas (NEVES, 2016).

Em relação a patogenia envolvida na amebíase, muito ainda necessitase ser elucidado, entretanto sabe-se que inicialmente há forte adesão da ameba a célula que será lesada. A adesão é propiciada por meio de lectinas presentes na superfície das amebas, logo em seguida tem-se a formação filopódios e fagocitose, respectivamente. Após a fagocitose do tecido, tem-se movimentos ameboides e a liberação de enzimas proteolíticas que possibilitam a progressão das amebas aos tecidos permitindo a invasão e destruição destes, sendo a enzima cisteína protease a principal responsável por lesões produzidas por E. histolytica. Além disso, as amebas patogênicas ainda podem fazer uso dos amebaporos, permitindo a indução de apoptose e lise osmótica das células-alvo (NEVES, 2016; NELSON; SINGH, 2018).

Mesmo a E.histolytica e E. dispar sendo morfologicamente iguais apresentam biologias distintas, uma vez que, a amebíase intestinal grave e extra-intestinal está presente em portadores de E. histolytica, enquanto que aqueles que estão contaminados por E. dispar apresentam-se assintomático ou com colites não-desentéricas (SILVA et al., 2013).

Em relação a sintomatologia e quadros clínicos, a amebíase se caracteriza por lesões ulcerativas e diarreia sanguinolenta, dor abdominal leve a moderada, possível diarreia intermitente alternando com prisão de ventre e tenesmo. A infecção pode ganhar nível sistêmico através da amebíase extraintestinal na qual pode-se observar o surgimento de abscessos hepáticos e pulmonares e outras complicações clínicas como colite necrótica fulminante e 
perfurações intestinais, sendo estas complicações a principal causa de morte nos casos de amebíase intestinal invasiva (NICHOLS, 2014).

Este protozoário pode ser detectado por meio de exame parasitológico de fezes, entretanto apresenta-se morfologicamente idêntico a Entamoeba dispar, contudo a pesquisa de coproantígenos através de ensaio de imunoadsorção enzimática (ELISA), imunofluorescência indireta, hemoaglutinação indireta e a reação em cadeira de polimerase (PCR) podem permitir diferenciá-las. Tais métodos, todavia têm o alto custo como fator limitante para sua utilização em grande escala nos serviços de saúde (ARROJA et al., 2010; SILVA et al., 2013).

O tratamento farmacológico deve ser empregado sempre que a presença de E. histolytica/E. dispar for comprovada nas fezes, mesmo que o paciente se apresente assintomático. Os medicamentos utilizados são o metronidazol ou tinidazol, entretanto se for identificado a presença de E. dispar nenhum tratamento precisa ser realizado, contudo nos países em desenvolvimento a falta de recursos dificulta a utilização métodos diagnósticos apropriados que permitam diferenciar estas duas amebas (NICHOLS, 2014; NELSON; SINGH, 2018). A profilaxia se dá através de saneamento básico, higiene pessoal, consumo de água e alimentos de boas procedência e tratamento dos infectados.

\subsection{Giardíase e Giardia lamblia}

A giardíase é uma protozoose causada pelo agente etiológico Giardia lamblia que é responsável por acometer cerca de 280 milhões de pessoas anualmente (EINARSSON; MA'AYEH; SVÄRD, 2016).

Giardia lamblia pode se apresentar na forma de cisto e de trofozoíto. O cisto é a forma infectante adquirida principalmente através de alimentos e água contaminados, possui cerca de $7 \mu \mathrm{m}$ de largura, $10 \mu \mathrm{m}$ de comprimento e de dois a quatro núcleos. Na região do citoplasma apresenta axóstilos, vacuolos, ribossomos, corpos parabasais e fragmentos do disco suctorial. Enquanto que o trofozoíto é a forma móvel, apresenta de $10 \mu \mathrm{m}$ de largura e $15 \mu \mathrm{m}$ de comprimento e uma região dorsal e outra ventral. Na região dorsal é possível observar dois axóstilos centrais e corpos parabasais, já na região ventral tem- 
se o disco adesivo e as enzimas tubucina e a giardina que permitem com que 0 processo de adesão a mucosa intestinal seja efetuado. Além disso, apresenta flagelos na região ântero-posterior e dois núcleos (HOOSHYAR et al., 2019; LEUNG et al., 2019).

A infecção por Giardia é iniciada pela ingestão de cistos que são estimulados por meio do ácido estomacal, bile e tripsina, permitindo a completa liberação do trofozoíto na região intestinal. Os trofozoítos se ligam ao epitélio intestinal por meio do disco adesivo permitindo assim danos teciduais. Devido a modificações ambientais na região intestinal não elucidadas, o trofozoíto passa pelo processo de encistamento, voltando novamente a ser cisto para posterior liberação ao meio juntamente com as fezes (EINARSSON; MA'AYEH; SVÄRD, 2016).

Em relação a patogenia, o parasita se replica sobre as células do epitélio intestinal podendo causar o processo de atapetamento e utiliza-se do disco adesivo para permitir a fixação e lesões mecânicas nas microvilosidades intestinais. Além disso, acredita-se que substâncias liberadas pelo parasito e a resposta inflamatória mediada pela imunidade do hospedeiro, possam contribuir para significativas modificações morfológicas do epitélio intestinal. Desta forma, o somatório de todos estes fatores, contribuem para o desenvolvimento da má absorção. Contudo, foram elucidadas algumas importantes informações acerca da interação entre as células epiteliais intestinais do hospedeiro e o trofozoíto de Giardia. Sabe-se que esse parasito é responsável pela liberação de algumas enzimas durante a interação com as células, como arginina deiminase, enolase e ornitina carbamoil transferase. A liberação de arginina deiminase, impossibilita que os enterócitos possam produzir o óxido nítrico, que por sua vez, é citotóxico para o parasito, além disso, as proteases de cisteína secretadas pela Giardia logo após o processo interação, possibilitam a clivagem de IL-8, reduzindo assim a infiltração de neutrófilos e dificultando a expulsão desse parasito do tecido intestinal (EINARSSON; MA'AYEH; SVÄRD, 2016; NEVES, 2016).

A giardíase pode se manifestar clinicamente na forma aguda ou crônica. A forma aguda é autolimitada durando cerca de duas semanas. Nesta fase, apresentam náuseas, anorexia, calafrios, febre, diarreia explosiva, perda de 
peso, vômito, flatulência e dor abdominal. Enquanto que na forma crônica, a duração é superior a um mês podendo a chegar a durar anos caso não haja tratamento. As manifestações clínicas mais comuns são episódios diarreicos contínuos, intermitentes ou esporádicos, anorexia, cefaleia, esteatorreia, mialgia e má absorção de lipídeos, proteínas, vitaminas lipossolúveis, vitamina B12 e ferro. Além de sérias comorbidades, como síndrome do intestino irritável, alergias alimentares, artrite e síndrome de fadiga crônica (EINARSSON; MA'AYEH; SVÄRD, 2016).

Para o diagnóstico pode-se utilizar ELISA, centrífugo-sedimentação e centrífugo-flutuação (cistos) (BICA; DILLENBURG; TASCA, 2011; BERNE et al., 2014) e método direto, para fezes diarreicas a fim de detectar o trofozoíto. O tratamento pode se dar por meio dos nitroimidazóis, principalmente 0 metronidazol e tinidazol. O correto tratamento de água, correta higienização de alimento, boa higiene pessoal e saneamento básico, são formas preventivas para evitar o desenvolvendo dessa enfermidade.

\section{Conclusão}

A ascaridíase, himenolepíase, amebíase e giardíase têm se apresentado como um importante problema de saúde pública no Brasil e no mundo, entretanto a melhoria do saneamento básico, higiene pessoal e dos alimentos e no tratamento da água podem contribuir para a diminuição da cadeia de transmissão. Além disso, o presente estudo pode servir para embasar outras pesquisas que tenham as parasitoses intestinais como foco centralizador e também atuar como possível material didático para a atualização de profissionais da saúde.

\section{Referências}

ANDRADE, Elizabeth Campos et al. Parasitoses intestinais: uma revisão sobre seus aspectos sociais, epidemiológicos, clínicos e terapêuticos. Revista APS, Juiz de Fora, MG, v.13, n.2, abr.jun, 2010. Disponível em:< https://periodicos.ufif.br/index.php/aps/article/view/14508>. Acesso em: 21 mai. 2020. 
ARROJA, Bruno et al. Invasive amebiasis. Jornal Português de Gastrenterologia, Lisboa, $\quad$ v.17, $\quad$ n.6, $2010 . \quad$ Disponível em:< http://www.scielo.mec.pt/scielo.php?script=sci abstract\&pid=S0872-

81782010000600004\&lng=pt\&nrm=iso $>$. Acesso em: 21 mai. 2020.

BELO, Vinícius Silva et al. Fatores associados à ocorrência de parasitoses intestinais em uma população de crianças e adolescentes. Revista Paulista de Pediatria, São Paulo, v.30, n.2, 2012. Disponível em:< https://www.scielo.br/pdf/rpp/v30n2/07.pdf $>$. Acesso em: 22 mai. 2020.

BERNE, Ana Cristina et al. Giardia lamblia: diagnóstico com o emprego de métodos microscópicos e Enzyme-linked immunosorbent assay (ELISA). Revista de Patologia Tropical, Goiânia, v.43, n.4, 2014. Disponível em:< http://repositorio.furg.br/bitstream/handle/1/7058/33602-141421-1-

PB.pdf?sequence=1>. Acesso em: 22 mai. 2020.

BICA, Vinícius C; DILLENBURG, Alexandre Francisco; TASCA, Tiana. Diagnóstico laboratorial da giardiose humana: comparação entre as técnicas de sedimentação espontânea em água e de centrífugo-flutuação em solução de sulfato de zinco. Revista do Hospital das Clínicas de Porto Alegre, Porto Alegre, v.31, n.1, 2011. Disponível em:< https://seer.ufrgs.br/hcpa/article/view/16962>. Acesso em: 22 mai. 2020.

BORGES, Wanessa Ferreira; MARCIANO, Franciele Maia; OLIVEIRA, Heliana Batista. Parasitos intestinais: elevada prevalência de Giardia lamblia em pacientes atendidos pelo serviço público de saúde da região sudeste de Goiás, Brasil. Revista de Patologia Tropical, Goiânia, v. 40, n. 2, p. 149-157, 2011.

BOURÉE, Patrice. Infecciones parasitarias del intestino.Tratado de medicina, v.18, n.3, p.1-8, 2014.

BRASIL. Ministério da Saúde. Doenças Infecciosas e Parasitária: Guia de Bolso. 8. ed. Brasília: Ministério da Saúde, 2010.

CAVALCANTE, Uluanna Maria Bastos; MELO, Silvia Adelaide Linhares de; LIMA, Caliandra Maria Bezerra Luna. Enteroparasitoses na população infantil, sua prevalência e os modelos de decisão utilizados: revisão sistemática. Revista Saúde e 
Pesquisa, Maringá, v.8, n.3, 2015. Disponível em:< https://periodicos.unicesumar.edu.br/index.php/saudpesq/article/view/4497>. Acesso em: 22 mai. 2020.

CHAVES, Antonio Carlos Palermo; SEIXAS FILHO, José Teixeira de; DANTAS, Marcia Machado Lima. Revisão do mecanismo fisiopatológico da amebíase. Revista Augustus, Rio de Janeiro, v.14, n.29, 2010. Disponível em:< http://www.educadores.diaadia.pr.gov.br/arquivos/File/2010/artigos teses/2011/ciencia s/09revisao mecanismo fisiopatologico amebiase.pdf >. Acesso em: 22 mai. 2020.

CHIEFFI, Pedro Paulo. Helmintoses e alterações ambientais e climáticas. Arquivos Médicos, São Paulo, v.60, n.1, 2015. Disponível em:< http://arquivosmedicos.fcmsantacasasp.edu.br/index.php/AMSCSP/article/view/163>. Acesso em: 22 mai. 2020.

DOLD, Christina; HOLLAND, Celia. Ascaris and ascariasis. Microbes and Infection, v.13, n.7, p.632-637, 2011.

EINARSSON, Elin; MA'AYEH, Showgy; SVÄRD, Staffan Gunnar. An up-date on Giardia and giardiasis. Corrent Opinioin in Microbiology, v.34, p.47-52, 2016.

FONSECA, Eduardo Oyama Lins et al. Prevalência e fatores associados às geohelmintíases em crianças residentes em municípios com baixo IDH no Norte e Nordeste brasileiros. Cadernos de Saúde Pública, Rio de Janeiro, v. 26, n.1, 2010. Disponível em:< https://www.scielo.br/scielo.php?pid=S0102-

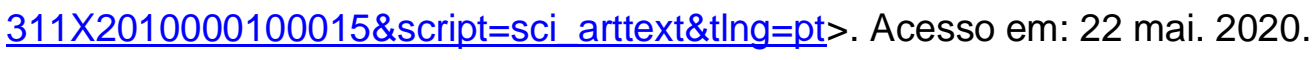

JOURDAN, Peter Mark et al. Soil-transmitted helmith infections. Lancet, v.391, p.252265, 2017. Disponível em:< https://www.thelancet.com/journals/lancet/article/PIIS01406736(17)31930-X/fulltext>. Acesso em: 22 mai. 2020.

HOOSHYAR, Hooshyar et al. Giardia lamblia infection: review of current diagnostic strategies. Gastroenterology and Hepatology from Bed to Bench, v.12, n.1, p.3-12, 2019.

LEUNG, Alexander K. C. et al. Giardiasis: An overview. Recent Patents on Inflammation \& Allergy Drug Discovery, v.13, n.2, p.134-143, 2019. 
LIMA, Caliandra Maria Bezerra Luna. Investigação de atividade antiparasitária do Allium sativum L. In vitro e In vivo. 2011. 90 f. Tese (Doutorado em Produtos Naturais e Sintéticos Bioativos) - Programa de Pós Graduação em Produtos Naturais e Sintéticos Bioativos, Universidade Federal da Paraíba, João Pessoa, 2011.

LIMA, Dayse da Silva et al. Parasitoses intestinais infantis no Nordeste brasileiro: uma revisão integrativa da literatura. Cadernos de Graduação - Ciências Biológicas e da Saúde Facipe, Recife, v.1, n.2, 2013. Disponível em:< https://periodicos.set.edu.br/index.php/facipesaude/article/view/1205/584>. Acesso em: 22 mai. 2020.

MAIA, Carlos Vangerre de Almeida; HASSUM, Izabella Cabral; VALLADARES, Gustavo Souza. Parasitoses intestinais em usuários do SUS em Limoeiro do Norte, Ceará, antes de expansão de sistema de esgotamento sanitário. Holos, Natal, v.2, n.31, $2015 . \quad$ Disponível em:< http://www2.ifrn.edu.br/ojs/index.php/HOLOS/article/view/1973>. Acesso em: 22 ma. 2020.

MENEZES, Saulo Almeida. et al. Epidemiologia do parasitismo provocado por Hymenolepis nana. In: MOSTRA CIENTÍFICA DE BIOMEDICINA, 01, 2016, Quixadá. Anais eletrônicos... Quixadá: Unicatólica, 2016.

NELSON, Joanna; SINGH, Upinder. Entamboeba histolytica (Amebiasis). Priciples and practice of pediatric infectious diseases. Philadelphia: Elsevier, 2018.

NEVES, David Pereira. Parasitologia Humana. 13. ed. São Paulo: Atheneu, 2016.

NICHOLS, Gordon L. Entamoeba histolytica. Encyclopedia of Food Safety, v.2, p. 31-36, 2014.

SALVADOR, Sibelle; STRECK, Emilio Luiz. Parasitoses em crianças: uma revisão bibliográfica dos casos na américa latina. Revista Inova Saúde, Criciúma, v.6, n.2, 2017. Disponível em:< http://periodicos.unesc.net//novasaude/article/view/3059>. Acesso em: 22 mai. 2020.

SILVA, Carinele Marques Veras et al. Contribuição ao estudo do diagnóstico clínico laboratorial e diferencial das Entamoeba histolytica e Entamoeba dispar. Scire Salutis, 
v.3, n.2, 2013. Disponível em:< https://www.sustenere.co/index.php/sciresalutis/article/view/ESS2236-

9600.2013.002.0009 >. Acesso em: 22 mai. 2020.

THOMPSON, Richard Christopher Andrew. Neglected zoonotic helminths: Hymenolepis nana, Echinococcus canadensis and Ancylostoma ceylanicum. Clinical Microbiology and Infection, v.21, n.5, p.426-432, 2015. 